\title{
Improving post-stroke recovery: the role of the multidisciplinary health care team
}

\author{
This article was published in the following Dove Press journal: \\ Journal of Multidisciplinary Healthcare \\ 22 September 2015 \\ Number of times this article has been viewed
}

\section{David J Clarke \\ Anne Forster}

Academic Unit of Elderly Care and Rehabilitation, Bradford Institute for Health Research, Bradford, UK
Correspondence: David J Clarke Academic Unit of Elderly Care and Rehabilitation, Bradford Institute for Health Research, Temple Bank House, Bradford Royal Infirmary, Duckworth Lane, Bradford BD9 6RJ, UK Tel +00 44 I 27438 344I/ +00 447503727990

Email d.j.clarke@leeds.ac.uk
Abstract: Stroke is a leading cause of serious, long-term disability, the effects of which may be prolonged with physical, emotional, social, and financial consequences not only for those affected but also for their family and friends. Evidence for the effectiveness of stroke unit care and the benefits of thrombolysis have transformed treatment for people after stroke. Previously viewed nihilistically, stroke is now seen as a medical emergency with clear evidence-based care pathways from hospital admission to discharge. However, stroke remains a complex clinical condition that requires health professionals to work together to bring to bear their collective knowledge and specialist skills for the benefit of stroke survivors. Multidisciplinary team working is regarded as fundamental to delivering effective care across the stroke pathway. This paper discusses the contribution of team working in improving recovery at key points in the post-stroke pathway.

Keywords: stroke care, rehabilitation, multidisciplinary, interdisciplinary, team working

\section{Introduction}

Stroke is an apt description of the disease as it occurs "at a stroke", the insult is immediate, and the effects may be prolonged with physical, emotional, social, and financial consequences not only for those affected but also for their family and friends. Despite advances in identification and reduction of risk, stroke remains a major illness. Annually, 17 million people worldwide suffer a stroke. Of these, 5 million die and another 5 million are left permanently disabled, placing a burden on family and community. ${ }^{1}$ At least 1.2 million people living in England have had a stroke, of whom 300,000 live with moderate-to-severe disability. ${ }^{2}$ Stroke is the leading cause of serious, long-term disability in the US. ${ }^{3}$ Stroke is an age-related condition, although people of any age can be affected. Approximately $26 \%$ of strokes occur in people below the age of $65^{2}$; it is estimated that 13 in 100,000 children suffer a stroke. ${ }^{4}$

The burden of stroke is considerable at a population, societal, and individual level. Costs are estimated at $£ 8$ billion per year in the UK, with $£ 3$ billion direct costs to the National Health Service, $£ 2.4$ billion in informal care costs, and $£ 1.8$ billion in lost productivity. ${ }^{5}$ Unplanned visits to the doctor and hospital readmissions contribute to the economic burden and cause stress and discomfort for patients. Post-stroke hospitalization rates are significantly higher than for a matched non-stroke cohort. ${ }^{6}$ One study reported that $<15 \%$ of surviving stroke patients had not been readmitted to hospital in 5 years. ${ }^{7}$ Cumulative risk of recurrent stroke at 10 years is $39 \%$. ${ }^{8}$ Evidence for the effectiveness of stroke unit care ${ }^{9}$ and the benefits of thrombolysis ${ }^{10}$ has transformed the treatment for stroke survivors. Previously viewed nihilistically, stroke is now seen 
as a medical emergency with clear evidence-based care pathways from hospital admission to discharge. However, despite these advances, longer term outcome remains poor for many, ${ }^{11-13}$ with unmet needs common. ${ }^{14}$ Prevalence of depression is $31 \%,{ }^{15}$ up to $40 \%$ of stroke survivors have loss of function of the upper limb at 1 year post-stroke, and $40 \%$ have problems with swallowing. A third of stroke survivors are aphasic, and $\sim 15 \%$ are incontinent at 1 year. ${ }^{2,5}$ Deficits in memory, attention and concentration, perception, spatial awareness (neglect), apraxia, and executive functioning are consequences of stroke. Many stroke survivors require assistance from informal caregivers, often family members, for activities of daily living, including bathing, dressing, and toileting. ${ }^{16}$ This burden of care has an important effect on caregivers' physical and psychosocial well-being, ${ }^{17,18}$ with up to $48 \%$ of caregivers reporting health problems, two-thirds a decline in social life, and high self-reported levels of strain. ${ }^{13}$

The evidence on which this paper draws is primarily of that presented in Cochrane and other systematic reviews published since 2000 and, where appropriate, evidence from quantitative and qualitative studies relevant to improving post-stroke recovery. The paper comments on contributions made by multidisciplinary teams (MDTs) in providing evidence-based care to improve post-stroke recovery. Individual responses to and recovery from neurological injury following stroke are complex and variable. This requires health professionals to work collaboratively to bring to bear their collective knowledge and specialist skills for the benefit of stroke survivors. In the discussion that follows, we first outline differences between multidisciplinary and interdisciplinary working to underline their contribution and use in stroke care. The paper is then divided into three sections to reflect the contribution of stroke teams at key points in the stroke pathway (Figure 1). These are first, the prehospital and emergency department (ED) period; second, the inpatient period; and third, the period after hospital discharge, including the related area of longer term support for stroke survivors.

\section{Multidisciplinary and interdisciplinary teams in stroke services}

MDT working is linked by policy makers and clinical guideline developers with improvements in the quality of stroke care. ${ }^{19-23}$ In the National Stroke Strategy for England, Wales, and Northern Ireland, ${ }^{22}$ six of 20 quality markers identified focus on coordinating rehabilitation professionals' specialist skills and knowledge to promote effective service delivery and improve patient outcomes. This confidence in the benefits accruing from MDT working stems partly from a Cochrane systematic review of trials of inpatient stroke care which found unequivocal evidence that organized inpatient care provided in stroke units by MDTs improved outcomes. ${ }^{9}$ This review of all previous randomized trials evaluating care in a stroke unit compared to treatment in a medical ward (21 trials, 3,994 participants) demonstrated that not only was mortality reduced but also patients in receipt of such care were also more likely to be independent and living at home 1 year after stroke. Advantages gained are persistent and applicable regardless of age, sex, disability, and level or type of stroke. ${ }^{9}$ The review authors explored features of stroke unit care provided in randomized trial evaluations and suggested that multidisciplinary rehabilitation, staff with a specialist interest in stroke or rehabilitation, and regular programs of staff education and training were key. Multidisciplinary rehabilitation is therefore now a central tenet of high-quality stroke care. ${ }^{5,19,22}$ However, the concepts of multidisciplinary and interdisciplinary working in stroke services are largely uncontested. ${ }^{20,21}$ The ways in which MDT working actually contributes to the improved outcomes seen in patients treated in stroke units has not been definitively established. A comment on team types is important before progressing to the discussion of
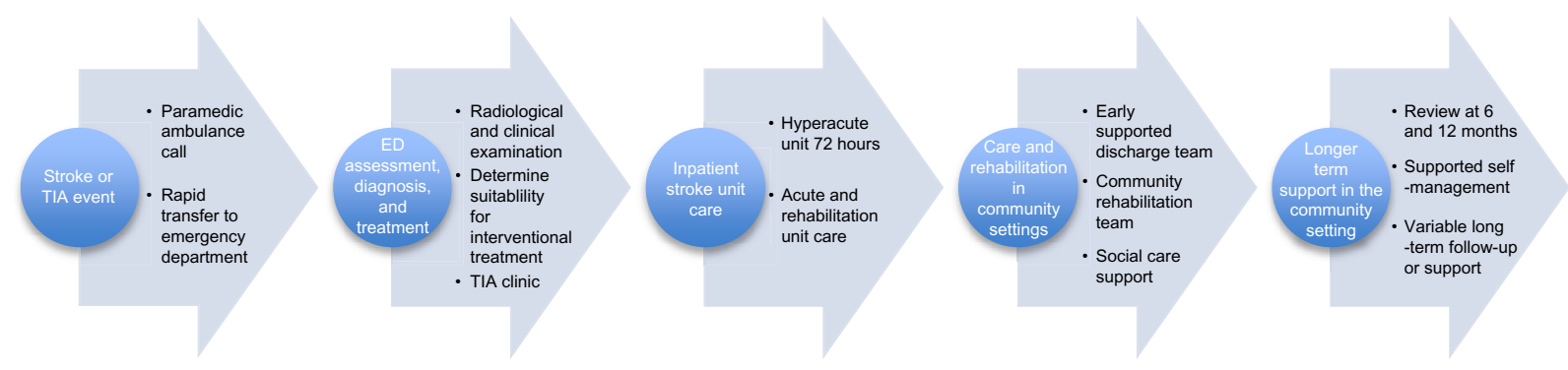

Figure I The stroke care pathway.

Abbreviations: TIA, transient ischemic attack; ED, emergency department. 
the contribution made by stroke teams in improving poststroke recovery.

An MDT is a collection of professionals from different disciplines who share a common area of working practice..$^{23,24}$ However, bringing individual professionals together does not automatically mean they will function cooperatively. ${ }^{25-27}$ There is commonly an organized division of labor in MDTs. Members share responsibility and accountability for patients' well-being, but typically, they each make and implement decisions independently and report on these to other MDT members. This can be an effective way to get individual disciplines' work done, but can mean that potential benefits of integrated team action are not realized. ${ }^{27-31}$

Stroke teams are larger than many health care teams, so coordination and effective collaboration are important. Interdisciplinary teamwork (IDT) implies that not only do team members perform activities toward a common goal, they also accept the added responsibility of group effort on behalf of patients. ${ }^{32,33}$ In IDTs, members contribute different professional perspectives, but goal setting, care planning, and decision-making are collaborative activities. In stroke services, this collaboration can occur in weekly, or more frequent, IDT meetings, ${ }^{19,32,34}$ and through ongoing patientfocused dialog..$^{32,33,35}$ Disciplinary articulation within IDTs is also important; here, team members develop understanding of each other's roles and recognize where overlap occurs. ${ }^{33,35,36}$ This understanding and acceptance of blurring of role boundaries facilitates rapid information exchange, enables early interventions, and underpins effective rehabilitation in secondary care, in early supported discharge (ESD) schemes and in longer term stroke care in the community settings. ${ }^{21,37,38}$ A more integrated and effective approach to working together is claimed for IDTs, ${ }^{32,33,35,36}$ which are more likely to be effective when team members function as equals, with respect for the skills and knowledge brought by each. However, there is evidence in stroke services and in health care more generally that this is easier to proclaim than to achieve..$^{21,25,30,31}$ We highlight in the inpatient stroke care section some of the ways in which reliance on MDT working may reduce the effectiveness of stroke teams.

\section{Recognition of stroke and transient ischemic attack}

One of the most significant changes in stroke care in the last 15 years has been the recognition that stroke-specific assessment and rapid transfer of people with stroke and transient ischemic attack (TIA) symptoms to ED saves lives and improves outcomes. ${ }^{5,19}$ This change was driven by the evidence that for those with ischemic stroke, intravenous thrombolysis is a proven treatment. ${ }^{10}$ Prehospital delay, ie, the delay between the symptom onset and seeking medical help, is the main contributor to suboptimal treatment for stroke. ${ }^{39-42}$ Recognition of stroke and TIA symptoms by members of the public is often poor, and coordination of Emergency Medical Services (EMS) and ED services, although improving, remains variable. ${ }^{39-45}$ Mass media campaigns, including in the UK, the Face, Arms, Speech, Time (FAST) campaign, had some effect in increasing public awareness of stroke and TIA but have largely been proven ineffective in changing the behavior. ${ }^{39,41-43}$ In the Barriers to the Early Assessment of TIA and Stroke (BEATS) study ${ }^{41}$ and in qualitative studies, ${ }^{42,46}$ reasons given for not seeking urgent medical attention included nonrecognition of symptoms, failure to appreciate the seriousness of symptoms, and uncertainty about the appropriateness of seeking urgent help, especially when symptoms were mild or transient. Time to specialist assessment is critical in reducing mortality and improving outcomes after stroke and TIA. In the Emergency Stroke Calls: Obtaining Rapid Telephone Triage (ESCORTT) ${ }^{40,45}$ study, researchers analyzed calls $(n=592)$ to EMS to determine the relationship between callers' description of potential stroke symptoms to EMS dispatchers and subsequent classification and prioritization of EMS responses. Jones et $\mathrm{al}^{40}$ reported that callers who identified the patient was having a stroke were correct in $89 \%$ of cases. Although, in common with the findings mentioned earlier, FAST test symptoms were mentioned in $<5 \%$ of calls, with falls and stroke being the most common statements made. At this critical time, it is essential that EMS dispatchers recognize stroke symptoms in callers' descriptions, which may be less than specific, and be able to coordinate team responses between EMS and the ED. Jones et $\mathrm{al}^{40}$ found that calls categorized as stroke by EMS dispatchers were commonly confirmed as stroke in the ED; they argued that further development and training of EMS and ED staff were needed to improve prehospital stroke recognition and expedite effective hyperacute stroke care. A coordinated and streamlined process that involves public education, education of EMS dispatchers, and collaboration between paramedic teams and stroke teams in EDs is essential. ${ }^{40,42,44,45}$ In addition to EMS and paramedic staff, hyperacute stroke teams must include a stroke physician, a stroke specialist nurse working closely with ED staff, and an imaging team led by a radiologist/neuroradiologist. Therefore, as a minimum, an MDT approach is fundamental to assessment and management of stroke and TIA in the ED. Written protocols delineating key stages in care increase the 
likelihood that stroke teams will provide effective hyperacute stroke management. ${ }^{19,47}$

\section{Hyperacute stroke care}

It is common for hyperacute stroke teams to be based on inpatient stroke units. Stroke physicians and stroke specialist nurses attend the ED when alerted by a Code: Stroke call. ${ }^{19,47}$ At the hyperacute end of the stroke pathway (Figure 1), stroke specialist nurses (alongside or prior to physician involvement) commonly take responsibility for patient assessment, including National Institutes for Health Stroke Scale scoring to assess stroke severity, instigating the diagnostic pathway, requesting computerized tomography (CT) scanning, and supporting treatment decision-making. Following rapid assessment by a stroke team in ED, the decision to provide thrombolysis normally requires $\mathrm{CT}$ or magnetic resonance imaging (MRI) of the brain, ideally completed immediately on arrival in ED and in all cases within 1 hour of arrival. ${ }^{19,44,47}$ Brain scanning ensures exclusion of hemorrhagic stroke, a prerequisite for thrombolysis administration. ${ }^{19,47}$ Thrombolysis is a reperfusion therapy designed to recanalize obstructed arterial vessels. Following arterial occlusion, two zones of local injury occur, the core and the ischemic penumbra. In the core, blood flow can be $<10 \%-25 \%$. This severe reduction in oxygen and glucose results in death of neurons and glial cells. However, cells within the ischemic penumbra remain viable for a short period of time as some oxygen and glucose are provided by collateral circulation. Intervening effectively in this time-sensitive period requires a high level of collaboration and shared understanding of progression in brain injury in hyperacute stroke teams; unless reperfusion is achieved, a wider area of infarction will develop and more severe disability and death is likely.

Thrombolysis is maximally effective within a narrow therapeutic window. Provided there are no contraindications, clinicians should consider administration of thrombolysis within 3 hours of known symptom onset for people of all ages. The Third International Stroke Trial ${ }^{48}$ indicated no increased risk to patients aged over 80 years during that time period, but that treatment efficacy reduced significantly after 3 hours. National Clinical Guidelines for Stroke ${ }^{19}$ recommend clinicians consider administration of intravenous thrombolysis between 3 hours and 4.5 hours for people under 80 . Between 3 hours and 6 hours, Intercollegiate Stroke Working Party $(\mathrm{p} 46)^{19}$ recommend clinicians review patients individually, but note that benefits of treatment with intravenous thrombolysis "are likely to be smaller than for those treated within
3 hours, but that the risk of worse outcomes including death will not on average be increased".

Collaborative, interdisciplinary team work, and a patientfocused organizational culture were found to be prominent features of hospitals achieving marked improvement and outstanding performance in door-to-balloon times for STsegment myocardial infarction. ${ }^{49}$ Fonarow et al ${ }^{50}$ suggested these elements may be particularly important in more complex clinical processes, such as door-to-needle time in acute ischemic stroke. In Finland, and later in Australia, Meretoja et al $^{51,52}$ demonstrated that a whole systems approach involving collaboration between health professionals can significantly improve the time between stroke recognition and transfer to ED, and once in ED, drive down the "doorto-needle time" for intravenous thrombolysis administration. Meretoja et $\mathrm{al}^{51,52}$ achieved reductions from $>60$ minutes and $>40$ minutes to as little as 20-25 minutes in Finland and Australia, respectively. The approach, known as the Helsinki model, ${ }^{51}$ is based on 12 linked components and takes a systems analysis and patient-focused approach to removing unnecessary barriers to rapid specialist assessment, brain imaging, and, where appropriate, thrombolysis. In common with the ESCORTT study, ${ }^{40,45}$ the Helsinki model targets education of EMS and paramedic teams in stroke recognition. The model goes further in actively demonstrating to these team members how their involvement in the model directly influences patients' experience in the ED and subsequent outcomes. Central to the model's effectiveness is a collaborative IDT approach whereby team members understand the interdependence of each others' role and contribution, and share responsibility for removal of organizational and professional barriers to service improvements. ${ }^{51,52}$ Removing barriers included developing protocols where instead of paramedics simply alerting the ED stroke team that a patient was en route to ED, call alert systems were changed to allow patient details to be shared prior to ED arrival so the team know who the patient is and can access their medical history as well as history gathered by the EMS team. Further changes required collaboration between imaging and stroke team members and included direct patient transfer to CT (instead of an ED stretcher) and immediate on-table CT interpretation by the stroke team. Such changes challenge existing organizational processes and also staff who are accustomed to working in particular ways, especially where out-of-hours services require revision to provide 24-hour CT and stroke team services rather than on-call services. While the Helsinki model has not yet achieved widespread adoption, Meretoja et $\mathrm{al}^{51,52}$ demonstrate that skilled leadership and an approach 
based on integrated IDT principles can achieve substantive change in short periods of time. Most importantly, in terms of improving patient outcomes, in the Australian center, $65 \%$ of patients are now treated within 60 minutes of ED arrival, this compares favorably with the reported average of $27 \%$ in US centers ${ }^{50}$ and $56 \%$ in the UK centers. ${ }^{34}$

\section{New options for treating ischemic stroke}

Recent research has demonstrated that mechanical clot retrieval can improve outcomes for those who have experienced an ischemic stroke caused by occlusions in the proximal anterior cerebral circulation. ${ }^{53-56}$ Results from four randomized controlled trials (RCTs) found improved functional independence at 3 months, with no significant increase in mortality in those treated using mechanical clot retrieval devices when compared to standard treatment. ${ }^{53-56}$ Currently, intra-arterial clot retrieval is only performed in specialist neurological centers. This intervention may become a mainstream therapeutic technique in the near future and therefore will require close collaboration between stroke teams and interventional radiologists.

\section{Inpatient stroke unit care}

The aforementioned developments have led to improvements in prehospital and hyperacute stroke care. However, a relatively small proportion of the total stroke population will benefit from intravenous thrombolysis or mechanical clot retrieval. The majority of stroke survivors will rely on rehabilitation provided by IDTs in stroke units and/or ESD teams. ${ }^{19}$ Inpatient stroke teams are usually based on designated stroke units and include stroke physicians, nurses, physiotherapists (PTs), occupational therapists (OTs), speech and language therapists (SALTs), and health care and therapy assistants. Therapy assistants are trained to support PTs or OTs; generic rehabilitation assistants have training to support PTs, OTs, SALTs, and nurses. In some hospitals, stroke coordinators (nurses or therapists) are core team members and work with stroke survivors, their families, and the wider stroke team from admission to discharge. Along with physicians and other stroke team members, stroke coordinators provide secondary stroke prevention and behavior change advice aimed at helping stroke survivors reduce the risk of recurrent events. Stroke physicians focus on medical management and oversee patient care from admission through to follow-up at 6 weeks; most advocate for and contribute fully to MDT or IDT working. In the UK, postdischarge patient follow-up is expected at 6 weeks and 6 months. ${ }^{19,34}$
In most National Health Service settings, dieticians, clinical psychologists, and social workers are not unit or ESD teambased and work more on the periphery of stroke teams. Early assessment of patients, normally within 24 hours, and appropriate referral prompted by National Clinical Guidelines ${ }^{19}$ and Sentinel Stroke National Audit Programme ${ }^{34}$ standards ensure that these professionals contribute to rehabilitation where specific needs are identified.

Stroke units include hyperacute units where stroke survivors undergo intensive physiological monitoring and medical stabilization during the first 72 hours post-stroke, and acute and rehabilitation units (often combined) where the remainder of the inpatient stay is experienced. To achieve reductions in mortality and increased independence associated with organized inpatient stroke care, stroke survivors should be admitted directly to stroke units from the ED and remain there for the duration of the inpatient stay. ${ }^{9}$ Currently, in England, $\sim 60 \%$ are admitted to a stroke unit within 4 hours of arrival in ED and up to $85 \%$ of all stroke survivors spend $>90 \%$ of their stay in a stroke unit. ${ }^{34}$

In most countries, inpatient stroke rehabilitation is underpinned by evidence-based National Clinical Guidelines ${ }^{19,57-59}$ and relies on a coordinated team approach to planning, delivering, and evaluating care. High-quality Cochrane systematic review evidence is available in relation to the types and effectiveness of some, but not all, rehabilitation interventions used by PTs, OTs, and SALTs working with stroke survivors. These interventions, although primarily managed by single disciplines, rely upon all members of stroke unit having an understanding of the principles underpinning the intervention and providing a rehabilitation-focused environment in which stroke survivors are encouraged or supported to continue therapeutic activity. Langhorne et $\mathrm{al}^{60}$ argued that interventions demonstrating most evidence of potential to improve outcomes were repetitive task-oriented training that targets activities or goals relevant to stroke survivors' identified needs and constraint-induced movement therapy. Cochrane reviews report that physiotherapy, occupational therapy, and speech and language therapy interventions can improve outcomes for stroke survivors, but the active ingredient and the form of intervention that is most effective have not been identified. Legg et al, ${ }^{61}$ in a review of nine RCTs $(1,258$ participants) found that OT increased personal activity of daily living (ADL) scores and reduced the odds of a poor outcome. For every eleven patients receiving an OT intervention to facilitate personal ADLs, one was spared a poor outcome.

Brady et $\mathrm{al}^{62}$ in a review examining speech and language therapy for aphasia after stroke included 39 RCTs 
(2,518 participants). The review identified some evidence of the effectiveness of SALT in improving functional communication and receptive and expressive language for people experiencing aphasia after stroke. However, Brady et $\mathrm{al}^{62}$ concluded that the evidence was not sufficient to conclude one specific SALT intervention was more effective than another. Similarly, in a larger review of approaches to physical rehabilitation after stroke, Pollock et $\mathrm{al}^{63}$ included 96 studies (10,401 participants). They concluded that physical rehabilitation, comprising a selection of components from different approaches, is effective for recovery of function and mobility after stroke. No single approach was found to be any more (or less) effective in promoting recovery of function and mobility. Given these findings, it is important that stroke team members adopt a consistent and evidencebased approach to rehabilitation practices. All stroke team members can support stroke survivors in practices related to improving independence in ADLs, in increased function and mobility, and in communication. Intercollegiate Stroke Working Party ${ }^{19}$ (p:32) guidelines recommend that all members of a stroke service should

use an agreed consistent approach for each problem faced

by a patient, ensuring that the patient is given the same

advice and taught the same technique to ameliorate or

overcome it.

There is some evidence that stroke teams who have adopted an interdisciplinary approach are more likely to work in this way. Bernhardt et al, ${ }^{64}$ in a small observational study $(n=95)$, compared physical activity patterns of patients managed in a stroke unit with specified mobilization protocols in Trondheim, Norway, with those managed in five stroke units without mobilization protocols in Melbourne, Australia. Results indicated that although patients in Melbourne and Trondheim had similar baseline characteristics, those in Melbourne spent 21\% more time in bed and only $12.2 \%$ undertook moderate/high activity; this was significantly lower than the percentage in Trondheim where $23.2 \%$ undertook moderate/high activity $(P<0.001)$. In addition to the presence of mobilization protocols in Trondheim, observations indicated that while Trondheim patients had on average twice as many therapy sessions as those in Melbourne, there was no significant difference in the average number of minutes per session. ${ }^{64}$ Mobilization began in Trondheim in the first 24-48 hours after stroke and continued as an intensive rehabilitation program up until 2 weeks post-stroke. A notable difference between the two units was the involvement of registered nurses with a high level of education and training in stroke rehabilitation. The mobilization intervention occurred 24 hours per day led primarily by a nursing team that were highly involved with mobilization. In Trondheim, nurses spent $23.2 \%$ of the active day with patients (compared with $14.1 \%$ in Melbourne). A small follow-up study explored interdisciplinary interactions in interviews with staff in both units, concluding that greater integration and sharing of knowledge, skills, and roles within the Trondheim team was instrumental in improving patient outcomes. ${ }^{65}$ The Collaborative Evaluation of Rehabilitation in Stroke across Europe (CERISE) project ${ }^{66-69}$ was a multicenter, longitudinal cohort study comparing inpatient stroke care and recovery patterns between four European rehabilitation centers (one unit in the UK, Germany, Switzerland, and Belgium). The project's aim was to assess variation in motor and functional recovery patterns for 6 months after stroke. One of the CERISE studies, examining use of time by PTs and OTs, reported a higher level of nursing involvement in rehabilitation activity in the UK unit, but this did not demonstrate any improvement in motor and functional recovery. No comparisons were made, but it appears likely that the interdisciplinary approach evident in Trondheim was not a feature of the UK unit. The CERISE group, while drawing attention to variation in the organization of rehabilitation work between the four centers, for example, discrete disciplinary working practices between PTs and OTs, did not attribute these to differences in team working per se, but rather focused on the efficiency of the organization of rehabilitation services. ${ }^{66-69}$ In a qualitative observational study of team working in two rehabilitation stroke units, Clarke $^{33}$ found that a high level of IDT working had been achieved primarily through learning from and about each others' rehabilitation practice, while team members worked jointly with stroke survivors. Regular problem-oriented and opportunistic dialog between team members led to strokeand therapy-specific language being increasingly shared. Importantly, understanding the rationale underpinning the thinking and beliefs of different disciplines resulted in a change in thinking from "what am I trained to do (separately) as an OT or nurse" to "what does the patient need and how can this be provided collaboratively by the stroke team". The impact on rehabilitation-related activity was similar to that observed in Trondheim, ${ }^{64,65}$ with registered nurses consistently applying rehabilitation principles agreed with therapists across 24 hours each day. In an action research study, ${ }^{70}$ development of a community of practice in a large stroke unit in London was reported to be instrumental in building a committed team of stroke clinicians, which displayed all 
the characteristics of interdisciplinary working. This team was recognized nationally for their success in developing an evidence-based stroke service in which nurses as well as therapists were central to providing rehabilitation. ${ }^{70}$

However, not all stroke teams adopt an interdisciplinary approach to their work. A study of interprofessional teamwork across stroke pathways ${ }^{21}$ conducted with five stroke teams (three inpatient and two community based, $n=402$ participants) identified, through interviews, observations, and staff surveys, a number barriers to interdisciplinary working that impacted on patient outcomes and staff satisfaction with their work. The authors found that the larger the team, the more the likelihood of uniprofessional groupings occurring ${ }^{21}$ and that nationally audited performance targets $^{34}$ were a disincentive to interdisciplinary working. In contrast to the studies outlined earlier, Harris et $\mathrm{al}^{21}$ found that despite having most contact with patients and carers, nurses had least involvement in the stroke teams as a whole and integrated working was uncommon in these teams. Similarly, in a process evaluation of a pragmatic cluster RCT of a caregiver training intervention, marked variability in team working approach was observed in six of ten stroke units observed. ${ }^{26}$ This variability reflected the MDT to IDT continuum already described. In a secondary analysis comparing compliance with the compulsory elements of the caregiver training intervention with team type, it was noted that teams with a loose MDT affiliation were less compliant with intervention delivery than those with an IDT approach. ${ }^{26}$ In practical terms, teams with an IDT approach were more likely to see delivery of caregiver training as a shared responsibility, more likely to share information on caregiver training in team meetings, and more likely to check with other team members that training had been provided.

\section{Early supported discharge}

While treatment in a stroke unit is enshrined in the stroke care pathway, pressures on in patient services are such that the length of stay in such units is reducing. A form of postdischarge MDT rehabilitation was developed to reduce the length of stay in hospital and evaluated under the umbrella term of early supported discharge. This provides an intuitively attractive model of replicating the successful features of organized stroke unit care within a person's own home where there is the potential to address disability and enhance participation. A Cochrane review of stroke specialist ESD teams $^{71}$ (14 trials with 1,957 participants) reported that ESD increased the likelihood that patients will regain independence in activities that support daily living and resulted in fewer patients requiring long-term institutional care, for mild to moderately disabled stroke survivors. This reinforces findings from previous research that rehabilitation in the home is beneficial, ${ }^{72,73}$ as it facilitates greater emphasis on daily activities. As with the stroke unit, the "winning" ingredients of ESD are more challenging to pin down. Fisher et $\mathrm{al}^{37}$ used a modified Delphi approach with the original ESD trialists to identify key features of ESD provision. These included the recommendation that ESD teams should be multidisciplinary, led by a co-coordinator who facilitates weekly meetings, have key workers assigned to each patient, and be based in hospitals in order to play an active role in discharge planning. The focus in the identified key features of ESD services is primarily on the patient, with strategies of how to address (or measure) carer health status or quality of life not identified. In qualitative interviews, carers expressed the view that appropriate levels of training, both practical and emotional, and information had not been provided in an appropriate format. ${ }^{38}$ However, the wider context of the patient and their family must be considered by ESD team members; this includes addressing carer needs. Focus on patient needs alone can inadvertently lead to the neglect of carers, who may experience considerable and sustained burden and anxiety. ${ }^{17,18,74}$ Information provision remains a commonly reported unmet need. ${ }^{12-14,37,38}$ Stroke survivors and carers consistently report that they do not know enough about the mechanisms, cause, and consequence of stroke. It is difficult to know whether this is a true expression of lack of needed knowledge or a reflection of stroke survivors' and carers' continued post-stroke uncertainty. It is important that stroke teams have an information strategy, ensuring that messages to patients and their families are consistent and that not only basic information is provided but also that they have the knowledge of where and how to access further information if required. Many stroke survivors experience a poor transition of care from hospital to home; the memory of this transition can influence their recovery. ${ }^{11}$ In the early weeks after stroke, the needs of stroke survivors are varied and complex and successfully addressing these (usually impairment based) needs requires expertise from a range of stroke specialist health professionals, as found in ESD teams. Inpatient and ESD or community-based stroke teams must work collaboratively to ensure that the patient and carer are fully informed of what services (if any) are provided postdischarge, that these services are similarly informed about patients' needs, and that any aids and appliances are provided in a timely manner. Patients and carers should also 
be made aware of services (eg, stroke clubs and respite care) and benefits that are available to them.

\section{Longer term stroke care}

It has to be recognized that input from specialist stroke teams cannot continue indefinitely. There is little current information on how best to provide longer term support for stroke survivors and their carers, although their needs have been identified. ${ }^{14}$ Expert consensus ${ }^{37}$ is that following input from a specialist stroke team, once rehabilitation goals have been met, the treatment plan may then be progressed by nonspecialist, community-based teams. This reflects a view that the stroke team contribution will vary according to patient need, and that later in the stroke recovery pathway, teams require knowledge and understanding of community-based services rather than specialist stroke knowledge. ${ }^{37}$ The need for some form of longer term follow-up has been acknowledged by the recommendation in the UK that all stroke survivors should be reviewed at 6 months, ${ }^{19,22}$ although the context and delivery of these reviews are unspecified and implementation is currently patchy. ${ }^{34}$

Self-management is increasingly regarded as an appropriate way to support people with long-term conditions ${ }^{75}$ and is now part of approaches to help people adjust to life after stroke. This adjustment takes time, and therefore supported self-management approaches may be more appropriately adopted in the later phase of stroke care. It is important that stroke teams consider how to support stroke survivors to make the transition toward a more self-management mode of care. ${ }^{76}$ Shared understanding is vital but needs work to achieve; research has indicated that lay people and health care professionals have different interpretations of self-management, with the former interpreting self-management in the context of their relationship with health professionals and the latter viewing self-management as a model of compliance. ${ }^{77}$ Greater consideration must be given to the nature of the relationship between patients and professionals in the context of both inpatient and community settings in order to determine how, through their interactions across the post-stroke pathway, stroke survivors and their families can be supported in becoming less dependent on professionals and more able to understand and manage their own condition.

\section{Conclusion}

Collaboration between health professionals is required across the entire stroke pathway. Although the terms are not often differentiated, there is a difference between multidisciplinary team and IDT working in stroke care. This paper has highlighted how these differences may impact both positively and negatively upon the care received by stroke survivors and their families and on the experience of staff working in stroke teams. Collaboratively, team working, particularly that which adopts an interdisciplinary approach, is a key contributor to care quality in stroke services. Stroke remains a complex and challenging condition, but there have been sustained improvement in early recognition, in hyperacute care, and in inpatient stroke unit and ESD services. These improvements now need to be matched by increasing focus on longer term support for stroke survivors. The international commitment to evidence-based services in stroke care suggests this can be achieved in due course.

\section{Disclosure}

The authors report no conflicts of interest in this work.

\section{References}

1. Feigin VL, Forouzanfar MH, Krishnamurthi R, et al; on behalf of the Global Burden of Diseases, Injuries, and Risk Factors Study 2010 (GBD 2010) and the GBD Stroke Experts Group. Global and regional burden of stroke during 1990-2010: findings from the Global Burden of Diseases Study 2010. Lancet. 2014;383(9913):245-255.

2. Stroke Association, UK. State of the Nation. Stroke Statistics. [Document on the Internet]; 2015. Available from: https://www.stroke.org.uk/ resources/state-nation-stroke-statistics. Accessed March 23, 2015.

3. Centers for Disease Control and prevention. Stroke Facts. [Document on the Internet]; 2014. Available from: http://www.cdc.gov/stroke/facts. htm. Accessed November 23, 2014.

4. Mallick A, O'Callaghan F. The epidemiology of childhood stroke. Eur J Paediatr Neurol. 2010;14(3):197-205.

5. National Audit Office. Progress in Improving Stroke Care. London: The Stationery Office Limited; 2010.

6. Lakshminarayan K, Schissel C, Anderson DC, et al. Five-year rehospitalization outcomes in a cohort of patients with acute ischemic stroke: Medicare linkage study. Stroke. 2011;42(6):1556-1562.

7. Bravata D, Ho S, Meehan T, Brass L, Concato J. Readmission and death after hospitalization for acute ischemic stroke: 5-year follow-up in the Medicare population. Stroke. 2007;38(6):1899-1904.

8. Mohan K, Wolfe C, Rudd A, Heuschmann P, Kolominsky-Rabas P, Grieve A. Risk and cumulative risk of stroke recurrence: a systemic review and meta analysis. Stroke. 2011;42(5):1489-1494.

9. Stroke Unit Trialists' Collaboration. Organised inpatient (stroke unit) care for stroke. Cochrane Database Syst Rev. 2013;(9). [Art No: CD000197].

10. Wardlaw JM, Murray V, Berge E, del Zoppo GJ. Thrombolysis for acute ischaemic stroke. Cochrane Database Syst Rev. 2014;(7). [Art No: CD000213].

11. Murray J, Young J, Forster A. Review of longer-term problems after a disabling stroke. Rev Clin Gerontol. 2007;17(04):277-292.

12. Hare R, Rogers H, Lester H, McManus R, Mant J. What do stroke patients and their carers want from community services? Fam Pract. 2006;23(1):131-136.

13. Salter K, Hellings C, Foley N, Teasell R. The experience of living with stroke: a qualitative meta-synthesis. J Rehabil Med. 2008;40(8): 595-602.

14. McKevitt C, Fudge N, Redfern J, et al. Self-reported long-term needs after stroke. Stroke. 2011;42(5):1398-1403.

15. Hackett M, Pickles K. Part I: frequency of depression after stroke: an updated systematic review and meta-analysis of observational studies. Int J Stroke. 2014;9:1017-1025. 
16. Guidetti S, Andersson K, Andersson M, Tham K, Von Koch L. Clientcentred self-care intervention after stroke: a feasibility study. Scand J Occup Ther. 2010;17(4):276-285.

17. Rigby H, Gubitz G, Phillips S. A systematic review of caregiver burden following stroke. Int J Stroke. 2009;4(4):285-292.

18. Greenwood N, Mackenzie A, Cloud GC, Wilson N. Informal carers of stroke survivors-factors influencing carers: a systematic review of quantitative studies. Disabil Rehabil. 2008;30(18):1329-1349.

19. Intercollegiate Stroke Working Party. National Clinical Guidelines for Stroke. 4th ed. London: Royal College of Physicians; 2012.

20. Clarke DJ. The role of multidisciplinary team care in stroke rehabilitation. Prog Neurol Psychiatry. 2013;17(4):5-10.

21. Harris R, Sims S, Hewitt G, et al. Interprofessional Teamwork Across Stroke Care Pathways: Outcomes and Patient and Career Experience. Final Report. London: NIHR service Delivery and Organisation Programme; 2013.

22. Department of Health. National Stroke Strategy. London: HMSO; 2007.

23. Reeves S, Lewin S, Espin S, Zwarenstein M. Interprofessional Teamwork for Health and Social Care. Chichester: Wiley Blackwell; 2010.

24. Payne M. Teamwork in Multiprofessional Care. Basingstoke: McMillan; 2000.

25. Miller C, Ross N, Freeman M. Interprofessional Practice in Health and Social Care. London: Arnold; 2001

26. Clarke DJ, Hawkins R, Sadler E, et al. Introducing structured caregiver training in stroke care: findings from the TRACS process evaluation study. BMJ Open. 2014;4:e004473.

27. Hewitt G, Sims S, Greenwood N, Jones F, Ross F, Harris R. Interprofessional teamwork in stroke care: is it visible or important to patients and carers? J Interprof Care. 2014;28(6):501-506.

28. Morris R, Payne O, Lambert A. Patient, carer and staff experience of a hospital based stroke service. Int J Qual Health Care. 2007;19: 105-112.

29. Suddick KM, de Souza LH. Therapists' experiences and perceptions of teamwork in a neurological rehabilitation: critical happenings in effective and ineffective teamwork. J Interprof Care. 2007;21:669-686.

30. Atwal A, Caldwell K. Do all health and social care professionals interact equally: a study of interactions in multidisciplinary teams in the United Kingdom. Scand J Caring Sci. 2005;19(3):268-273.

31. Baxter SK, Brumfitt SM. Benefits and losses: a qualitative study exploring healthcare staff perceptions of team working. Qual Saf Health Care. 2008;17:127-130.

32. McCallin AM, McCallin MCH. Factors affecting successful team working and strategies to facilitate successful collaborative teamwork NZ J Physiother. 2009;37:62-68.

33. Clarke DJ. Achieving teamwork in stroke units: the contribution of opportunistic dialogue. J Interprof Care. 2010;24(3):285-297.

34. Stroke Sentinel National Audit Programme Summary Report October to December 2014. England: Royal College of Physicians; 2015.

35. McCallin AM. Pluralistic dialoguing: a theory of interdisciplinary team working. Grounded Theory Rev. 2004;4(1):25-42.

36. Mandy P. Interdisciplinary rather than multidisciplinary or generic practice. Brit J Ther Rehabil. 1996;3:110-112.

37. Fisher RJ, Gaynor C, Kerr M, et al. A consensus on stroke early supported discharge. Stroke. 2011;42:1392-1397.

38. Cobley CS, Fisher RJ, Chouliara N, Kerr M, Walker MF. A qualitative study exploring patients' and carers' experiences of Early Supported Discharge Services after stroke. Clin Rehabil. 2013;27(8): 750-757.

39. Dombrowski SU, White M, Mackintosh JE, et al. The stroke 'Act FAST' campaign: remembered but not understood? Int J Stroke. 2015;10(3):324-330.

40. Jones S, Carter B, Ford GA, et al. The identification of acute stroke: an analysis of emergency calls. Int J Stroke. 2013;8(6):408-412.

41. Wilson AD, Coleby D, Taub NA, Weston C, Robinson TG. Delay between symptom onset and clinic attendance following TIA and minor stroke: the BEATS study. Age Ageing. 2014;43(2):253-256.
42. Jones S. Acute stroke and the public's response to symptoms, Doctoral Thesis, University of Central Lancashire, 2013.

43. Lecouturier J, Rodgers H, Murtagh MJ, White M, Ford GA, Thomson RG. Systematic review of mass media interventions designed to improve public recognition of stroke symptoms, emergency response and early treatment. BMC Public Health. 2010;10:784.

44. Lin CB, Peterson ED, Smith EE, et al. Emergency medical service hospital pre-notification is associated with improved evaluation and treatment of acute ischemic stroke. Circ Cardiovasc Qual Outcomes. 2012;5:514-522.

45. Watkins CL, Jones S, Leathley MJ, et al. Emergency Stroke Calls: Obtaining Rapid Telephone Triage (ESCORTT) - A Programme of Research to Facilitate Recognition of Stroke by Emergency Medical Dispatchers. Vol 2. London: Programme Grants for Applied Research; 2014.

46. Mikulik R, Bunt L, Hrdlicka D, Dusek L, Vaclavik D, Kryza J. Calling 911 in response to stroke: a nationwide study assessing definitive individual behaviour. Stroke. 2008;39(6):1844-1849.

47. Alberts MJ, Latchaw RE, Jagoda A, et al; Brain Attack Coalition; for the Brain Attack Coalition. Revised and updated recommendations for the establishment of primary stroke centers: a summary statement from the brain attack coalition. Stroke. 2011;42:2651-2665.

48. The IST-3 collaborative group. The benefits and harms of intravenous thrombolysis with recombinant tissue plasminogen activator within $6 \mathrm{~h}$ of acute ischaemic stroke (the Third International Stroke Trial [IST-3]): a randomised controlled trial. Lancet. 2012;379(9834): 2352-2363.

49. Bradley EH, Nallamothu BK, Herrin J, et al. National efforts to improve door-to-balloon time results from the Door-to-Balloon Alliance. $J \mathrm{Am}$ Coll Cardiol. 2009;54(25):2423-2429.

50. Fonarow GC, Smith EE, Saver JL, et al. Improving door-to-needle times in acute ischemic stroke. The design and rationale for the American Heart Association/American Stroke Association's target: stroke initiative. Stroke. 2011;42:2983-2989.

51. Meretoja A, Strbian B, Mustanoja S, Tatlisumak T, Lindsberg PJ, Kaste M. Reducing in-hospital delay to 20 minutes in stroke thrombolysis. Neurology. 2012;79(4):306-313.

52. Meretoja A, Weir L, Ugalde M, et al. Helsinki model cut stroke thrombolysis delays to 25 minutes in Melbourne in only 4 months. Neurology. 2013;81(12):1071-1076.

53. Berkhemer OA, Fransen PS, Beumer D, et al; MR CLEAN Investigators. A randomized trial of intraarterial treatment for acute ischemic stroke. N Engl J Med. 2015;372:11-20.

54. Goyal M, Demchuk AM, Menon BK, et al; ESCAPE Trial Investigators. Randomized assessment of rapid endovascular treatment of ischemic stroke. N Engl J Med. 2015;372:1019-1103.

55. Campbell BC, Mitchell PJ, Kleinig TJ, et al; EXTEND-IA Investigators. Endovascular therapy for ischemic stroke with perfusion-imaging selection. N Engl J Med. 2015;372:1009-1018.

56. Saver JL, Goyal M, Bonafe A, et al; SWIFT PRIME Investigators. Stent-retriever thrombectomy after intravenous t-PA vs t-PA alone in stroke. N Engl J Med. 2015;372(24):2285-2295.

57. National Stroke Foundation. Clinical Guidelines for Stroke Management 2010. Melbourne Australia. [Online]. Available from: http://strokefoundation.com.au/health-professionals/tools-and-resources/clinicalguidelines-for-stroke-prevention-and-management/. Accessed March 6, 2015.

58. Jauch EC, Saver JL, Adams HP Jr, et al; on behalf of the American Heart Association Stroke Council, Council on Cardiovascular Nursing, Council on Peripheral Vascular Disease, and Council on Clinical Cardiology. Guidelines for the early management of patients with acute ischemic stroke: a guideline for healthcare professionals from the American Heart Association/American Stroke Association. Stroke. 2013;44:870-947.

59. Heart and Stroke Foundation, Canada. Canadian Stroke Best Practice Recommendations. 2015 Canada. [Online]. Available from: http:// www.strokebestpractices.ca/. Accessed March 18, 2015. 
60. Langhorne P, Bernhardt J, Kwakkel G. Stroke rehabilitation. Lancet. 2011;377:1693-1702.

61. Legg L, Drummond A, Leonardi-Bee J, et al. Occupational therapy for patients with problems in personal activities of daily living after stroke: systematic review of randomised trials. BMJ. 2007;335:922.

62. Brady MC, Kelly H, Godwin J, Enderby P. Speech and language therapy for aphasia following stroke. Cochrane Database of Syst Rev. 2012;5. [Art No: CD000425]

63. Pollock A, Baer G, Campbell P, et al. Physical rehabilitation approaches for the recovery of function and mobility following stroke. Cochrane Database Syst Rev. 2014;4. [Art No: CD001920].

64. Bernhardt J, Chitravas N, Meslo IL, Thrift AG, Indredavik B. Not all stroke units are the same: a comparison of physical activity patterns in Melbourne, Australia, and Trondheim, Norway. Stroke. 2008;39(7):2059-2065.

65. Purvis T, Bernhardt J, Indredavik B, Cadilhac DA. Interdisciplinary team interactions in stroke units: can team dynamics influence patient outcomes from a clinician's perspective. Int J Phys Med Rehabil. 2014;:S3:007.

66. De Wit L, Putman K, Dejaeger E, et al. Use of time by stroke patients. A comparison of four European rehabilitation centers. Stroke. 2005;36: 1977-1983.

67. De Wit L, Putman K, Lincoln N, et al. Stroke rehabilitation in Europe. What do physiotherapists and occupational therapists actually do? Stroke. 2006;37:1483-1489.

68. Putman K, De Wit L, Schupp W, et al. Use of time by physiotherapists and occupational therapists in a stroke rehabilitation unit: a comparison between four European rehabilitation centres. Disabil Rehabil. 2006;28: $1417-1424$.
69. De Wit L, Putman K, Schuback B, et al. Motor and functional recovery after stroke: a comparison of 4 European rehabilitation centers. Stroke. 2007;38:2101-2107.

70. Kilbride C, Perry L, Flatley M, Turner E, Meyer J. Developing theory and practice: creation of a Community of Practice through Action Research produced excellence in stroke care. J Interprof Care. 2011; 25(2):91-97.

71. Langhorne P, for the Early Supported Discharge Trialists. Services for reducing duration of hospital care for acute stroke patients (review). Cochrane Database Sys Rev. 2005;(2):CD000443.

72. Gladman JR, Lincoln NB, Barer DH. A randomised controlled trial of domiciliary and hospital-based rehabilitation for stroke patients after discharge from hospital. J Neurol Neurosurg Psychiatry. 1993;56(9): 960-966.

73. Young JB, Forster A. The Bradford community stroke trial: results at six months. BMJ. 1992;304(6834):1085-1089.

74. Jaracz K, Grabowska-Fudala B, Górna K, Jaracz J, Moczko J, Kozubski W. Burden in caregivers of long-term stroke survivors: prevalence and determinants at 6 months and 5 years after stroke. Patient Educ Couns. 2015;98(8):1011-1016.

75. Corben S, Rosen R. Self-Managementfor Long-Term Conditions: Patients' Perspectives on the Way Ahead. London: King's Fund; 2005.

76. de Silva D. Evidence: Helping People Help Themselves: A Review of the Evidence Considering Whether it is Worthwhile to Support SelfManagement. London: The Health Foundation; 2011.

77. Sadler E, Wolfe CDA, McKevitt C. Lay and health care professional understandings of self-management: a systematic review and narrative synthesis. Sage Open Med. 2014;2.
Journal of Multidisciplinary Healthcare

\section{Publish your work in this journal}

The Journal of Multidisciplinary Healthcare is an international, peerreviewed open-access journal that aims to represent and publish research in healthcare areas delivered by practitioners of different disciplines. This includes studies and reviews conducted by multidisciplinary teams as well as research which evaluates the results or conduct of such teams or

\section{Dovepress}

healthcare processes in general. The journal covers a wide range of areas and welcomes submissions from practitioners at all levels, from all over the world. The manuscript management system is completely online and includes a very quick and fair peer-review system. Visit http://www.dovepress.com/testimonials.php to read real quotes from published authors. 